\title{
Prácticas de estudiantes universitarios relacionados con la alimentación de sus hijos preescolares
}

\section{Practices of college students related to the feeding of their preschool children}

\section{RESUMEN}

Introducción: Diferentes estilos de crianza pueden o no, favorecer en los niños conductas saludables sobre la alimentación. La restricción, presión para comer, y el uso de alimentos como control comportamental, generan respuestas negativas hacia los alimentos. El enrolamiento positivo, puede favorecer una alimentación saludable. La adopción de un estilo de crianza particular puede estar mediado por factores demográficos y socioeconómicos. Objetivo: Identificar las prácticas parentales de estudiantes universitarios relacionados con la alimentación de sus hijos, y evaluar si hubo diferencias según características socio-demográficas y económicas. Metodología: Se realizó un estudio de corte transversal analítico en 43 estudiantes de pregrado de una universidad pública colombiana, con hijos en edad preescolar y convivencia permanente. Se identificó el estilo parental mediante el cuestionario de prácticas parentales alimentarias -Parental Feeding Practices-. Se analizó la edad, nivel e ingreso socio económico, el peso y talla del estudiante universitario, entre otros. Resultados: Las prácticas de los estudiantes se orientaron al enrolamiento positivo. La edad y factores de tipo demográfico y socioeconómico determinaron la orientación respecto al tipo de práctica. Conclusión: Este estudio proporciona información sobre el estilo de educación en relación con algunos factores demográficos y socioeconómicos en una población poco explorada, que servirá de referencia para futuros estudios.

Palabras clave: Alimentación; Escolares; Estilos de crianza; Padres universitarios; Prácticas parentales; Preescolares.

\footnotetext{
ABSTRACT

Introduction: Different parenting styles may or may not favor healthy eating behaviors in children. Restriction, pressure to eat, and the use of food for behavioral control generate negative responses to food. Positive role modeling can promote a healthy diet. The adoption of a particular parenting style may be mediated by demographic and socioeconomic factors. Objective: We identified the parenting practices of college students related to the feeding of their children and evaluated if there were differences according to socio-demographic and economic characteristics. Methodology: We conducted an
}

Edna Magaly Gamboa-Delgado ${ }^{1}$, Claudia Amaya Castellanos², Gloria Esperanza Prada Gómez'.

\author{
1. Escuela de Nutrición y Dietética \\ Universidad Industrial de Santander, \\ Bucaramanga, Colombia. \\ 2. Escuela de Medicina, Departamento de Salud Pública, \\ Universidad Industrial de Santander, \\ Bucaramanga, Colombia.
}

Dirigir correspondencia a: Edna Magaly Gamboa Delgado.; Escuela de Nutrición y Dietética. Universidad Industrial de Santander. Teléfono: (57) 76344000 Ext. 3229.

Email: magalygamboa@yahoo.com; emgamboa@uis.edu.co

Este trabajo fue recibido el 13 de abril de 2018. Aceptado con modificaciones: 28 de junio de 2018. Aceptado para ser publicado: 09 de agosto de 2018.

analytical cross-sectional study in 43 undergraduate students of a Colombian public university with children of preschool age and permanent cohabitation. Parenting style was identified using the Parental Feeding Practices questionnaire. Age, socioeconomic level and income, weight and height of the university student, among others, were analyzed. Results: The students' practices were oriented to positive role modeling. Age, demographic and socioeconomic factors related to type of parenting practice. Conclusion: This study provides information on the style of upbringing in relation to some demographic and socioeconomic factors in a population little explored, which will serve as a reference for future studies. Keywords: College parents; Feeding; Parental practices; Parenting styles; Preschoolers; Schoolchildren. 


\section{INTRODUCCIÓN}

Diferentes estilos de crianza o prácticas parentales pueden favorecer o no en los niños, conductas saludables en torno a la alimentación. Estos estilos, son prácticas educativas parentales que determinan tipos de educación familiar específica'. Algunos autores señalan, que además de las prácticas de los progenitores, se incluyen también las actitudes, y que éstas en su conjunto proporcionan el soporte emocional en el que los comportamientos de los padres son expresados e interpretados por los niños ${ }^{2}$. En este sentido es claro que la crianza repercute en la alimentación de los menores'.

Dentro de los tipos de prácticas más utilizadas en la alimentación de los preescolares se encuentran la restricción, la presión para comer, y el uso de alimentos como control del comportamiento ${ }^{3,4}$. Sin embargo, estas prácticas para algunos autores pueden tener problemas al generar efectos contrarios. Por ejemplo, la restricción, la cual por lo general se orienta a limitar alimentos considerados poco saludables como dulces o golosinas ${ }^{5}$, puede aumentar el deseo de comer. Por su parte, ejercer presión, generalmente hacia alimentos saludables ${ }^{5}$, puede generar respuestas afectivas de tipo negativo hacia los alimentos, lo que puede disminuir el gusto por los mismos ${ }^{6}$, e incluso llegar a inhibir el deseo, lo que concuerda con algunos estudios donde se ha identificado una relación directa entre exceso de peso y restricción, y bajo peso con presión parental ${ }^{7,8}$. Así mismo, utilizar un sistema de recompensa que premie o castigue el acto alimenticio, puede evitar la autorregulación sobre procesos como el hambre y la saciedad ${ }^{3}$, lo que a su vez impide la autonomía del individuo, al estar mediado únicamente por factores externos para lograr su acción y los efectos deseados.

Otras prácticas se han orientado a utilizar los propios alimentos como recompensa, e incluso se refieren prácticas donde la alimentación responde al manejo de respuestas emocionales, las cuales en ambos casos distan de ser prácticas de crianza saludables, al contribuir a la generación de patrones alimenticios negativos en los menores ${ }^{9}$. Un estudio realizado en Brasil en 2011, refiere que las madres entrevistadas, fueron conscientes de haber utilizado una o más estrategias inadecuadas en la alimentación de sus hijos: "cambalache, castigo, coerción, insistencia, "camuflaje", y juego y oferta solamente de los alimentos preferidos por los niños"10.

Sin embargo, las prácticas descritas anteriormente, aunque se identifican como estilos altamente utilizados por padres y cuidadores, si bien con diferencias culturales, no son los únicos. Existen igualmente prácticas positivas en torno a los procesos alimentarios. Uno de ellos es el modelamiento, a partir del cual los padres establecen una relación favorable hacia los alimentos saludables. Los comen de manera frecuente delante de sus hijos e igualmente verbalizan delante de estos, las bondades y lo agradable que resulta consumirlos ${ }^{11}$. De 32 estudios revisados sobre modelamiento en una revisión sistemática del año 2017, 28 presentaron asociaciones significativas, lo que sugiere que este tipo de práctica tiene un efecto importante sobre los comportamientos alimenticios de los niños ${ }^{11}$. En esta misma línea, pero enfocado directamente al consumo de frutas como alimento saludable, estudios concluyen, que el consumo de los padres es el predictor más importante del consumo infantil ${ }^{12}$.

Por su parte en el estudio realizado en Brasil y señalado anteriormente para mostrar prácticas negativas, algunas madres, en menor proporción, expresan haber utilizado estrategias adecuadas, como estimular el olfato y la manipulación de alimentos, al igual que motivar a probar y preparar alimentos que tienden a ser rechazados, utilizar el uso de la huerta casera como un elemento motivador para la siembra y consumo de alimentos y manejar un lenguaje dramático para mostrar las características de los alimentos a los niños ${ }^{10}$.

Existe escasa evidencia sobre la descripción de las prácticas parentales de estudiantes universitarios que ejercen su rol de padre o madre, los cuales por su edad, situación específica y nivel académico suponen estilos de crianza particulares. Es por ello que el presente estudio busca determinar la prevalencia de las prácticas parentales relacionadas con la alimentación de sus hijos en estudiantes universitarios y evaluar las diferencias de éstas según características demográficas y socio económicas de los estudiantes.

\section{MATERIAL Y MÉTODOS Tipo de estudio y Criterios de elegibilidad}

Un estudio de corte transversal descriptivo fue realizado en estudiantes universitarios. Se incluyó en el estudio a estudiantes de pregrado de una universidad pública ubicada en el nororiente colombiano, que tuviesen hijos en edad pre escolar y que convivieran con ellos, de manera permanente, al momento de la encuesta. La muestra correspondiente a los estudiantes analizados representa a los estudiantes de pregrado de esta universidad que cumplen con los mismos criterios de inclusión empleados en el estudio.

\section{Variables}

La variable dependiente de este estudio estuvo conformada por las prácticas parentales, de los estudiantes universitarios, relacionadas con la alimentación de sus hijos. Estas fueron determinadas mediante la aplicación del cuestionario de prácticas parentales alimentarias (Parental Feeding Practices, PFP, por sus siglas en inglés) ${ }^{9}$. Este cuestionario ha sido validado en población mexicanaamericana y sus resultados indican buena validez inicial y reproducibilidad. Los resultados de ese proceso de validación se realizaron a través de análisis de factores confirmatorio que identificó cuatro dimensiones de las prácticas parentales relacionadas con la alimentación de sus hijos: enrolamiento positivo, presión para comer, uso de alimentos para control de comportamiento y restricción de la cantidad de alimentos. En cuanto a la consistencia interna, medida a través del Alfa de Cronbach, se alcanzaron coeficientes, para la mayoría de factores de primer orden, entre 0,55 y 0,90, con un valor promedio de 0,73 y entre 
0,70 y 0,91 para los factores de segundo orden, con valor promedio de $0,81^{9}$. Cada pregunta de este cuestionario, tiene opciones de respuesta en una escala de 1: nunca, 2: algunas veces, 3: seguido, 4: muy seguido y 5 : siempre, representando de menor a mayor la frecuencia usual con la que los padres realizan cada práctica.

Además, se analizaron variables como edad (en años cumplidos), nivel académico o semestre (número del semestre académico en el cual estaban matriculados), facultad o área de estudio (nombre de la facultad a la que pertenece la carrera que estaban cursando), nivel socio económico (bajo, medio o alto), ingresos económicos (ingresos económicos inferiores o no a un salario mínimo mensual), estado civil (soltero (a), casado (a), unión libre, separado/divorciado(a), viudo(a)), ser cabeza de familia (si/no), número de hijos (número total de hijos), peso (kilogramos) y talla (centímetros) del estudiante universitario.

\section{Proceso de recolección de datos}

Se realizó una convocatoria masiva utilizando las redes sociales de la universidad, a través de los centros de estudios de cada una de las carreras de pregrado y con el apoyo de la División de Bienestar Universitario. También se realizó búsqueda activa de los estudiantes que fuesen padres o madres a través de invitación personalizada en los salones de clase de cada facultad. Esta convocatoria, al igual que las mediciones, fue realizada por estudiantes de último semestre académico de Nutrición y Dietética previamente capacitadas y entrenadas bajo técnicas estandarizadas.

\section{Análisis Estadístico}

Se realizó un análisis descriptivo de las variables de interés en el cual las variables categóricas fueron presentadas como porcentajes, y las variables continuas como mediana, y su respectivo rango o su rango intercuartil. Para evaluar las diferencias de las prácticas parentales, según características demográficas y socio económicas de los estudiantes universitarios, se usaron pruebas de Wilcoxon Rank-sum (Mann-Whitney). Todas las pruebas estadísticas fueron consideradas a dos colas, tomando como estadísticamente significativos a los valores de $\mathrm{p}>0.05$. Todos los datos fueron analizados en el programa estadístico Stata 12.0 (College Station, TX: Stata Corporation).

\section{Consideraciones éticas}

El protocolo de este estudio fue aprobado por el comité de ética en investigación científica de la universidad donde se realizó la investigación. Todos los participantes firmaron el consentimiento informado.

\section{RESULTADOS}

\section{Características demográficas y socio económicas de los estudiantes universitarios}

Se analizó una muestra de 43 estudiantes universitarios que son padres o madres de niños (as) en edad preescolar. La distribución de los estudiantes según la facultad a la que pertenecen o área de estudio al interior de la universidad fue: facultad de ciencias humanas $(37,21 \%)$, facultad de ingenierías físico mecánicas $(23,26 \%)$, facultad de salud (20,93\%), facultad de ingenierías físico químicas (11,63\%) y facultad de ciencias $(6,98 \%)$.

El $33,33 \%$ era cabeza de familia y $86,05 \%$ de los participantes en el estudio tenía solo un hijo (a) y sobre su ocupación, 44,19\% de los estudiantes manifestó que solo estudia, mientras un $41,86 \%$ estudia y trabaja y $13,95 \%$ además de estudiar, recibe algún tipo de remuneración a través auxiliaturas estudiantiles.

Los ingresos mensuales, en la mayoría de los casos, es inferior a un salario mínimo mensual legal vigente (SMMLV) $(60,98 \%)$.

\section{Prácticas de cuidado y relaciones parentales}

$\mathrm{Al}$ indagar sobre la cantidad de tiempo que los padres dedican a compartir con sus hijos en actividades como leer, comer, jugar, dialogar o hacer tareas, en promedio, éstos dedican 20 horas de lunes a viernes (Desviación Estándar: D.E) 11,16 horas, rango: de 1 a 48 horas) y 15 horas los fines de semana (D.E 14,78, rango de 0 a 64 horas). Sobre la persona encargada del cuidado de los niños la mayor parte del tiempo, quienes ejercen ese rol en su mayoría, son la madre $(27,91 \%$ de lunes a viernes y $44,19 \%$ los fines de semana), madre y padre $(18,60 \%$ de lunes a viernes y $34,88 \%$ los fines de semana), abuelos $(16,28 \%$ de lunes a viernes) y sólo el padre (13,95\% los fines de semana).

Tabla 1. Características sociodemográficas de los estudiantes universitarios

\begin{tabular}{|lcc|}
\hline Características & n o Mediana & (\%) o [R.I] \\
\hline Edad (años) & 23 & {$[3.0]$} \\
Sexo & & \\
Femenino & 36 & $(83.72)$ \\
Masculino & 7 & $(16.28)$ \\
& & \\
Nivel Socio económico & & \\
Bajo & 20 & 46.51 \\
Medio y medio alto & 23 & 53.49 \\
& & \\
Semestre académico & & 32.56 \\
De ${ }^{\circ}$ a 5 ${ }^{\circ}$ nivel & 14 & 67.44 \\
De 6 ${ }^{\circ}$ a 10 & \\
Estado Civil & 29 & 44.19 \\
Soltero & & 18.60 \\
Casado & & 32.56 \\
Unión Libre & 19 & 4.65 \\
Separado/Divorciado & 2 & {$[7.38]$} \\
Índice de Masa Corporal & 22.38 & \\
(kg/m ${ }^{2}$ ) & & \\
R.I: Rango Intercuartil & & \\
& &
\end{tabular}




\section{Comportamientos alimentarios en la familia}

Las personas que suelen acompañar, con mayor frecuencia, a los niños mientras consumen los alimentos, son padre y madre (44.19\%) o sólo la madre (37.21\%). Sobre la conducta habitual de los niños a la hora de comer, $57.14 \%$ de los padres expresó que sus hijos comen con entusiasmo, mientras $21.43 \%$ rechaza la comida y $11.90 \%$ juega con la comida. Cuando los niños rechazan la comida, el comportamiento más común de los padres fue motivarlo a comer $(53.13 \%)$.

Los participantes del estudio indicaron que cuando motivan a los niños a comer, las estrategias usadas con mayor frecuencia son emplear juegos y palabras (39.53\%, $34.88 \%$, respectivamente) y en menor proporción gestos, regalos y otros $(4.65 \%, 9.30 \%$ y $11.63 \%$, respectivamente).

Todos los encuestados manifestaron que mientras el niño (a) está comiendo ellos se dedican a comer con éstos y no realizan otro tipo de actividad.

\section{Prácticas Parentales relacionadas con la alimentación de sus hijos}

Las prácticas más comunes de los estudiantes, relacionadas con la alimentación de sus hijos, fueron preguntar al niño (a) por lo que él/ella come y monitorear o limitar los alimentos con alto contenido de calorías, al igual que animar o felicitar por el consumo de una alimentación saludable, todas ellas pertenecientes a la dimensión de enrolamiento positivo de los padres en la alimentación de los niños (as) (Tabla 2). A su vez, las prácticas menos frecuentes fueron animar a los niños a comer menos, de la dimensión restricción de cantidad de alimentos, seguida por usar alimentos para controlar las emociones del niño (a), de la dimensión uso de alimentos para controlar comportamientos de los niños (Tabla 2).

\section{Diferencias en cuanto a las Prácticas Parentales relacionadas con la alimentación de sus hijos, según características demográficas y socioeconómicas}

En cuanto a diferencias estadísticamente significativas de las prácticas parentales, según las características de interés, se encontró que el monitoreo o limitación de alimentos con alto contenido de calorías fue diferente según la edad de los padres (Mediana en quienes tienen 23 años de edad o menos: 3.7, rango: 2.6 a 4.6 vs Mediana en personas con 24 años o más: 4.4, rango 2.9 a 5.0; $p=0.004$ ). A su vez, animar por el uso de nuevos y variados alimentos fue diferente según el nivel socio económico (Mediana en estudiantes de nivel socio económico medio: 4.0, rango: 2.0 a 5.0 vs Mediana en personas de nivel socio económico bajo: 3.0, rango 1.5 a 5.0; $\mathrm{p}=0.046$ ), así como preguntar al niño (a) por lo que él/ella come (Mediana en estudiantes de nivel socio económico medio: 5.0, rango: 1.0 a 5.0 vs Mediana en personas de nivel socio económico bajo: 3.7, rango 2.5 a $5.0 ; p=0.014$ ).

Tabla 2. Prácticas Parentales Relacionadas con la Alimentación de sus Hijos

\section{Prácticas}

Dimensión: Enrolamiento positivo en la alimentación de los niños (as)

Monitorear/limitar alimentos con alto contenido de calorías

Animar/Felicitar por alimentación saludable

Animar por el uso de variedad de nuevos alimentos

Preguntar al niño (a) por lo que él/ella come

Proveer porciones de alimentos pequeñas

Dimensión: Presión para comer

Decir al niño (a) que coma todo lo del plato

Requerir que el niño (a) coma aún si no tiene hambre
Mediana

Rango

Dimensión: Uso de alimentos para controlar comportamientos de los niños (as)

Usar de alimentos para controlar emociones

Usar de alimentos como recompensa

Dimensión: Restricción de cantidad de alimentos

Animar a los niños (as) a comer menos

Permitir al niño (a) controlar el snacking y segundas porciones

Limitar comer entre comidas

Permitir que el niño (a) deje la mesa sin terminar de comer
1.0 a 4.5 
Respecto a proveer porciones pequeñas de alimentos a sus hijos, se evidenciaron diferencias según la presencia de exceso de peso (Mediana en personas sin sobrepeso u obesidad: 2.5 , rango de 1.5 a 3.5 vs personas con éste evento: 3.0, rango de 1.5 a 5.0; $p=0.047$ ).

Tanto para el uso de alimentos como recompensa, como para limitar que los niños coman entre los tiempos de comida principales (desayuno, almuerzo y cena) se encontraron diferencias según la edad (Mediana en quienes tienen 23 años de edad o menos: 2.5, rango: 1.0 a 3.5 vs Mediana en personas con 24 años o más: 1.7, rango 1.0 a 3.2; $p=0.015$ y Mediana en quienes tienen 23 años de edad o menos: 2.3, rango: 1.0 a 4.0 vs Mediana en personas con 24 años o más: 2.6 , rango 2.3 a 4.6; $p=0.018$, respectivamente).

\section{DISCUSIÓN}

Este artículo se enfoca hacia un aspecto fundamental en el proceso de alimentación de los niños, tal como lo son las prácticas o estilos de crianza que utilizan sus padres, específicamente relacionadas con el abordaje que hacen respecto a la nutrición de sus hijos. Este aspecto resulta de particular relevancia en el contexto de los padres y madres universitarios, dado que el tener un hijo (a) durante la etapa universitaria puede considerarse un factor determinante del trayecto de vida en la medida que se aumentan sus responsabilidades, se debe asumir una serie de roles asociados con la adultez y aumenta la demanda de autonomía para el sostenimiento personal y de los hijos, tanto como la toma de decisiones respecto al cuidado y la crianza. Todos estos aspectos de manera simultánea con la carga académica, se podrían traducir en falta de tiempo o de calidad para la dedicación a una crianza adecuada de sus hijos ${ }^{13}$.

Es así que se ha descrito que existe una influencia ambiental del control que ejercen los padres sobre los comportamientos alimentarios de sus hijos en la composición corporal de los niños en términos de su índice de masa corporal o de la prevalencia de exceso de peso ${ }^{14}$. Un estudio prospectivo en niños de 5 a 7 años de edad encontró que las actitudes alimentarias de los padres, respecto a responsabilidad percibida, predijo una reducción en el índice de masa corporal de los niños a los 7 años de edad. El estilo parental de monitoreo de la alimentación también predijo una disminución en el IMC de los niños, mientras estilos parentales de restricción de alimentos, predijo mayor $\mathrm{IMC}^{15}$. De esta manera, la evidencia sugiere que los padres pueden influenciar el peso de sus niños y es así como esta interacción padres-hijos debe ser considerada en más investigaciones al respecto que permitan una comprensión más amplia de este fenómeno para dar solución al problema del sobrepeso infantil a través de intervenciones efectivas.

Los resultados de este estudio señalan que las prácticas ejercidas con mayor frecuencia por los estudiantes universitarios entrevistados fueron las pertenecientes a la dimensión de enrolamiento positivo respecto a la alimentación de sus hijos, mientras las menos prevalentes corresponden a la dimensión de uso de alimentos para controlar el comportamiento de los niños (as). Al respecto, algunos estudios señalan que durante los primeros años de vida puede ser más benéfico el uso de un estilo parental autoritario, dado que este se asocia con un mejor consumo de verduras y frutas, aunque este debe estar mediado por la afectividad, al igual que debe incluir la estimulación, el modelamiento y la disponibilidad de frutas y verduras ${ }^{16}$, los cuales son claros elementos del enrolamiento positivo.

Aunque no se identificó ningún estudio relacionado específicamente con universitarios, la revisión señala que el nivel educativo, principalmente el de la madre, está asociado con el consumo de verduras, dado que estas tienden a estimular y alentar la ingesta en sus hijos, al igual que comer junto a ellos ${ }^{17}$, tal y como ocurre con los padres y madres de este estudio.

Adicionalmente, en cuanto a las diferencias respecto a esas prácticas según las demás características analizadas, monitorear o limitar los alimentos altamente calóricos entre los tiempos de comida principales, fueron mayores en los padres de mayor edad (24 años o más), lo que supondría, según autores como Birch y Betsell, un riesgo para generar conductas contrarias a las esperadas ${ }^{5,6}$.

Entre tanto, usar alimentos como recompensa fue una práctica más común entre los estudiantes de 23 o menos años de edad, lo que coincide con las prácticas que realizan muchos padres, poniendo en riesgo la autorregulación de los niños ${ }^{3}$.

En cuanto a la edad de la madre, estudios señalan que ésta puede funcionar como elemento protector para el desarrollo de hábitos alimentarios saludables y una dieta adecuada. Hijos de madres mayores tienden a consumir por ejemplo, más frutas y a tener un mayor equilibrio calórico y lipídico. Madres más jóvenes, son descritas como madres poco maduras, lo que según algunos autores podría representar un elemento a considerar para el desarrollo de sus hijos ${ }^{8}$.

Por otra parte, según el nivel socio económico, los estudiantes de nivel socioeconómico medio animan más el consumo de alimentos variados y preguntan más por lo que sus hijos comen comparado con sus compañeros de niveles económicos bajos. Nuestros hallazgos contrastan con estudios que revelan que madres de preescolares de bajos ingresos, utilizan diferentes tipos de prácticas para promover y restringir el consumo de alimentos, pero en su mayoría se caracterizan por ser estilos más autoritarios ${ }^{19}$.

Se ha descrito que este tipo de madres, además proporcionan ambientes estructurados a la hora de la alimentación, por ejemplo, sin la presencia de televisión o sentándose en familia para $\operatorname{comer}^{20}$. Esto coincide con estudios realizado en mujeres hispanas de bajos ingresos, en donde señalan que estas tienden a ser más controladoras, al utilizar prácticas restrictivas y a ejercer más presión sobre la alimentación de sus hijos, comparadas con aquellas madres de ingresos socioeconómicos medios ${ }^{21}$.

En este estudio también se halló que los estudiantes que se encontraban en sobrepeso u obesidad proveen porciones de alimentos más pequeñas a sus hijos que los 
que no presentaban estas condiciones. Al respecto, un estudio realizado con madres con trastornos alimentarios, señalan que se pueden disminuir los conflictos relacionados con las prácticas de alimentación de sus hijos, si estás no se centran ni median el proceso a partir de sus propias problemáticas ${ }^{22}$, lo que da sentido a los resultados encontrados en el presente estudio, aunque el sobrepeso y la obesidad no sean considerados como trastornos alimentarios. Sin embargo, estas interpretaciones difieren de otro estudio donde señalan la relación entre ambientes familiares obesigénicos, particularmente la presencia de los padres, con patrones de alimentación inadecuados en los niños, los cuales además se presentan con mayor fuerza si la familia pertenece a un contexto de bajos recursos ${ }^{23}$.

La mayoría de estudios se centran en la participación de las madres en las prácticas alimenticias, porque son ellas las que permanecen más tiempo con los hijos, y son ellas las que principalmente se encargan de la compra, preparación y alimentación de los niños, comparado con los padres, quienes tienden a involucrarse prioritariamente en las actividades de juego ${ }^{24}$, aunque se reconoce que la nueva generación de padres está teniendo una participación mayor en la alimentación de los hijos ${ }^{18}$. En este sentido, algunos autores señalan la importancia que los padres se involucren, ya que juegan un papel importante en la imposición de prácticas alimenticias de los menores ${ }^{25}$.

Las limitaciones de este estudio se relacionan con la baja cantidad de estudiantes universitarios que cumplían los criterios de inclusión y que por tanto participaron en el estudio, lo cual podría representar un bajo poder estadístico.

La principal fortaleza de este estudio consiste en el riguroso procedimiento de recolección de datos, el cual garantiza calidad del estudio y disminución de sesgos.

\section{CONCLUSIONES}

El estilo de crianza utilizado en la alimentación de los niños, aunque no es único, ni estandarizado, en el caso de padres universitarios está orientado a la estimulación y el reforzamiento positivo frente a conductas alimenticias saludables de sus hijos, lo que supone que el nivel educativo es un factor que favorece este estilo de parentalidad. Sin embargo, el estilo de crianza esta mediado por características de tipo socioeconómico. A mayor estrato de los universitarios, mayor presencia de este tipo de parentalidad. Son los cuidadores, principalmente la madre, la que estimula hábitos de alimentación saludables en sus hijos, aunque esto dependerá de la edad. Mamás universitarias muy jóvenes tenderán a prestarle menor interés a este aspecto.

Además de identificar las prácticas alimentarias de crianza y su relación con condiciones de tipo económico, social y demográfico, el mayor aporte de este estudio radica en visibilizar el fenómeno en una población poco explorada, lo que constituye una ventana de oportunidad para profundizar más en el tema. En este sentido, los resultados de este estudio podrían ser útiles para el diseño de políticas de primera infancia que involucren el apoyo a padres jóvenes respecto a estilos de crianza positivos para el bienestar y la salud de sus hijos.

\section{BIBLIOGRAFÍA}

1. Ventura A. Birch L. Does parenting affect children's eating and weight status?. Int J Behav Nutr Phys Act 2008; 5: 15.

2. Rhee K. Childhood overweight and the relationship between parent behaviors, parenting style and family functioning. Ann Am Acad Pol Soc Sci 2008; 615: 12-37.

3. Fisher JO. Birch LL. Restricting access to foods and children's eating. Appetite 1999; 32: 405-419.

4. Mitchel G. Farrow C. Haycraft E. Meyer C. Parental influences on children's eating behaviour and characteristics of successful parent-focussed interventions. Appetite 2012; 60(1): 85-94.

5. Birch L. L. Fisher J. O. Grimm-Thomas K. Markey C. N. Sawyer R. Johnson S. L. Confirmatory factor analysis of the Child Feeding Questionnaire. A measure of parental attitudes, beliefs and practices about child feeding and obesity proneness. Appetite 2001; 36: 201-210.

6. Batsell W. R. Jr. Brown A. S. Ansfield M. E. Paschall G. Y. "Youwill eat all of that!": a retrospective analysis of forced consumption episodes. Appetite 2012; 38: 211-219.

7. Gray WN. Janicke DM. Wistedt KM. Dumont-Driscoll MC. Factors associated with parental use of restrictive feeding practices to control their children's food intake. Appetite 2010; 55(2): 332-337.

8. Hurley KM. Cross MB. Hughes SO. A systematic review of responsive feeding and child obesity in high-income countries. I Nutr 2011; 141(3): 495-501.

9. Tschann J. Gregorich S. Penilla C. Pasch L. Cynthia L. de Groat C1, et al. Parental feeding practices in Mexican American families: initial test of an expanded measure. Int I Behav Nutr Phys Act 2013; 10(6): 1-11.

10. Sanches M, Andrade M. Perceptions and practices of Brazilian preschool 'mothers about feeding of their children. Enferm Glob 2011; 10(21): 1-11.

11. Yee $A$, Lwin $M$, Ho S. The influence of parental practices on child promotive and preventive food consumption behaviors: a systematic review and meta-analysis, Int I Behav Nutr Phys Act 2017; 14: 47.

12. Vereecken C, Rovner A, Maes L. Associations of parenting styles, parental feeding practices and child characteristics with young children's fruit and vegetable consumption. Appetite 2010; 55: 589-596.

13. Miller Arvizu V. Be a mother and a student. An exploration of characteristics of the college students with children and brief notes for your study. Rev Educ Sup 2016; 1(177): 17-42.

14. Afonso L, Lopes C, Severo M, Santos S, Real H, Durão C, Moreira $P$, Oliveira A. Bidirectional association between parental child-feeding practices and body mass index at 4 and 7 y of age. Am J Clin Nutr 2016; 103(3): 861-867.

15. Faith MS, Berkowitz RI, Stallings VA, Kerns J, Storey M, Stunkard AJ. Parental Feeding Attitudes and Styles and Child Body Mass Index: Prospective Analysis of a Gene-Environment Interaction. Pediatrics 2004;114 (4):.e429-e436.

16. Blissett, J. Relationships between parenting style, feeding style and feeding practices and fruit and vegetable consumption in early childhood. Apettite 2011; 57(3): 826-831.

17. Cooke L, Wardle J, Gibson E, Sapochnik M. Demographic, familial and trait predictors of fruit and vegetable consumption by pre-school children. Public Health Nutr 2003; 7(2): 295302. 
18. Navia B, Ortega $R$, Rodríguez-Rodríguez E, Aparicio A. Perea J.M. The mother's age as conditioning of food consumption and energy and nutrient intake of their children in preschool age. Nutr Hosp 2009; 24(4): 452-458.

19. Ventura A. Gromis J. Lohse B. Feeding Practices and Styles Used by a Diverse Sample of Low-income Parents of Preschool-age Children. J Nutr Educ Behav 2010; 42(4): 242-249.

20. Carnell S, Cooke L, Cheng R, Robbins A, Wardle J. Parental feeding behaviours and motivations: a qualitative study in mothers of UK pre-schoolers. Appetite 2011; 57(3): 665-673.

21. Worobey J, Borrelli A, Espinosa C, Worobey H. Feeding Practices of Mothers from Varied Income and Racial/Ethnic
Groups. Early Child Dev Care 2013; 183(11): 1661-1668.

22. Stein A, Woolley H, MCPherson K. Conflict between mothers with eating disorders and their infants during mealtimes. $\mathrm{Br}$ J Psychiatry 1999; 175: 455-461.

23. Pisabarrol R, Recalde A, Irrazábal E, Chaftare Y. ENSO children1: First national survey of overweight and obesity in Uruguayan children. Rev Méd Urug 2002; 18(3): 244-250.

24. Brent AM, Mills GA. Comparison of mother and father involvement with their preschool age children. Early Child Res Q 1993; 8(4): 457-477.

25. Johannsen D, Johannsen M, Speker B. Influence of Parents' Eating Behaviors and Child Feeding Practices on Children's Weight Status. Obesity (Silver Spring) 2006; 14(3): 431-439. 